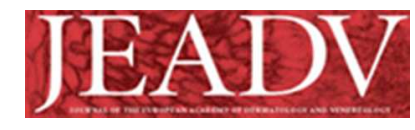

\title{
Peri-meatal PeIN and urethral SCC: a case report
}

\begin{tabular}{|r|l|}
\hline Journal: & Journal of the European Academy of Dermatology and Venereology \\
\hline Manuscript ID & JEADV-2017-0102.R1 \\
\hline Manuscript Type: & Letter to Editor \\
\hline Keywords: & $\begin{array}{l}\text { penile intraepithelial neoplasia, urethral squamous cell carcinoma, } \\
\text { cystoscopy }\end{array}$ \\
\hline \multicolumn{2}{|l}{} \\
\hline
\end{tabular}

SCHOLARONE ${ }^{m}$

Manuscripts 


\title{
Peri-meatal PeIN and urethral SCC: a case report
}

Word count: 529

Table count: 0

Figure count: 2

PR Doiron

Department of Dermatology, Chelsea and Westminster Hospital Chelsea and Westminster Hospital NHS Foundation Trust 369 Fulham Road, London, UK SW10 9NH

H du P Menage

Department of Dermatology, University Hospital Lewisham Lewisham and Greenwich NHS Trust

High Street, Lewisham, UK SE13 6LH

\author{
A Freeman \\ Department of Pathology, University College Hospital London \\ University College London Hospitals NHS Foundation Trust \\ 250 Euston Road, $3^{\text {rd }}$ Floor Central Wing \\ London, UK NW1 2PG \\ A Muneer \\ Dept of Urology and NIHR Biomedical Research Centre \\ University College London Hospitals NHS Foundation Trust \\ 250 Euston Road, $3^{\text {rd }}$ Floor Central Wing \\ London, UK NW1 2PG \\ CB Bunker - Corresponding Author \\ Department of Dermatology, University College Hospital London \\ University College London Hospitals NHS Foundation Trust \\ 250 Euston Road, $3^{\text {rd }}$ Floor Central Wing \\ London, UK NW1 2PG \\ chris.bunker@uclh.nhs.uk \\ Tel: 02034479224 \\ Fax: 02034479278 \\ Funding statement: None \\ Disclosures: None declared
}


A 55-year-old man presented with an asymptomatic lesion adjacent to the urethral meatus of one year's duration (Fig. 1). His medical history was significant for quiescent lung sarcoidosis (treatment never required), asthma and irritable bowel syndrome. His only medication was a budesonide/formoterol inhaler. The plaque had slowly been increasing in size, had not ulcerated or bled and had not impacted sexual or urinary function. Examination did not reveal extension into the urethra. Biopsy revealed undifferentiated penile intraepithelial neoplasia (PeIN) III/carcinoma in situ. Circumcision and simultaneously performed repeat glans biopsy revealed similar histopathology with no evidence of invasion. Koilocytic change was observed and immunostaining was positive for high-risk human papillomavirus (HPV) types 16 \& 18. Histopathology of the prepuce showed koilocytic change but no evidence of atypia. Residual glanular disease resolved following a 4-week course of 5-fluorouracil 5\% cream. No clinically obvious urethral extension or palpable lymphadenopathy was present at follow up visits. Eleven months after circumcision the patient reported a tender lump in the left groin. Examination revealed a firm, fixed slightly tender lymph node, with no contralateral lymphadenopathy. The glans and other genital skin was clear. Fine needle aspiration revealed metastatic squamous cell carcinoma (SCC). Cystoscopy showed a normal bladder and urethra up to the navicular fossa, where an area of aberrant mucosa was visualized. Biopsy and subsequent urethrectomy both showed extensive undifferentiated PeIN and focally invasive SCC (Fig. 2), again positive for high-risk HPV. MRI revealed pathological left inguinal and external iliac nodes. Subsequent inguinal and pelvic lymph node dissection revealed three positive nodes on the left 
side. Review of the two original glans biopsies did not reveal missed invasive disease. No alternative primary SCC was found on full skin examination or proctoscopy. Imaging did not reveal right sided disease. The patient is currently undergoing radiotherapy with adjunctive chemotherapy. Guidance regarding this particular clinical scenario is scarce in the medical literature. In one published case report of glanular PeIN extending into the external urethral orifice, the patient underwent a partial penectomy with a clear urethral margin, and no invasive disease was found ${ }^{1}$. In a series of five patients who underwent distal urethrectomy for penile CIS extending into the urethra, one was found to have concomitant glanular SCC 2 . A case of occult distal urethral SCC presenting as an enlarged lymph node has also been published ${ }^{3}$. Currently, no specific guidance exists regarding investigation of carcinoma in situ arising in the peri-meatal area ${ }^{4,5}$. Recent urethral carcinoma guidelines do not address screening for urethral disease in the context of known PeIN on the glans ${ }^{6}$. There are no data to suggest what proportion of men with peri-meatal PeIN will have urethral involvement. This case raises the important issue of considering cystoscopy to exclude potential contiguous or skip urethral lesions in peri-meatal PeIN, whether due to HPV or lichen sclerosus. Topical therapy alone may be inadequate for peri-meatal disease, and establishment of histologically negative margins by conventional surgical methods should be considered. The relationship between HPV and penile PeIN and SCC is well established $^{4,5}$, and may lead to non-contiguous disease of the anogenital skin. This case informs the debate about HPV vaccination programs in males in the prophylaxis and treatment of anogenital malignancy ${ }^{7}$. 


\section{References}

1 - Yasuda M, Tamura A, Shimizu A, Takahashi A, Ishikawa O. Bowen's disease involving the urethra. J Derm. 2005;32:210-213.

2 - Pedrosa JA, Amstutz SP, Bihrle R, Mellon MJ. Distal urethrectomy for localized penile squamous carcinoma in situ extending into the urethra: an update series. Int Uro Nephrol. 2014;46:1551-55.

3 - Hammer-Hansen N, Hoyer S, Jensen JB. Occult distal urethral carcinoma presenting as metastatic carcinoma in the inguinal lymph nodes. Scand J Uro. 2015;49(1):79-80.

4 - Hakenberg OW et al. EAU guidelines on penile cancer: 2014 update. Eur Uro. 2015;67:142-150.

5 - Morton CA, Birnie AJ, Eedy DJ. British Association of Dermatologists' guidelines for the management of squamous cell carcinoma in situ (Bowen's disease) 2014. Br J Dermatol. 2014;170:245-260.

6 - Gakis G, et al. EAU guidelines on primary urethral carcinoma. Eur Uro. 2013;64:823-30.

7 - Doiron PR, Bunker CB. Expanding the benefits of HPV vaccination to boys and men. The Lancet. 2016;388:659.

\section{Figure legend}

Figure 1: Peri-meatal erythematous plaque, histopathology confirmed penile intraepithelial neoplasia.

Figure 2: Invasive urethral squamous cell carcinoma (x40 magnification). 


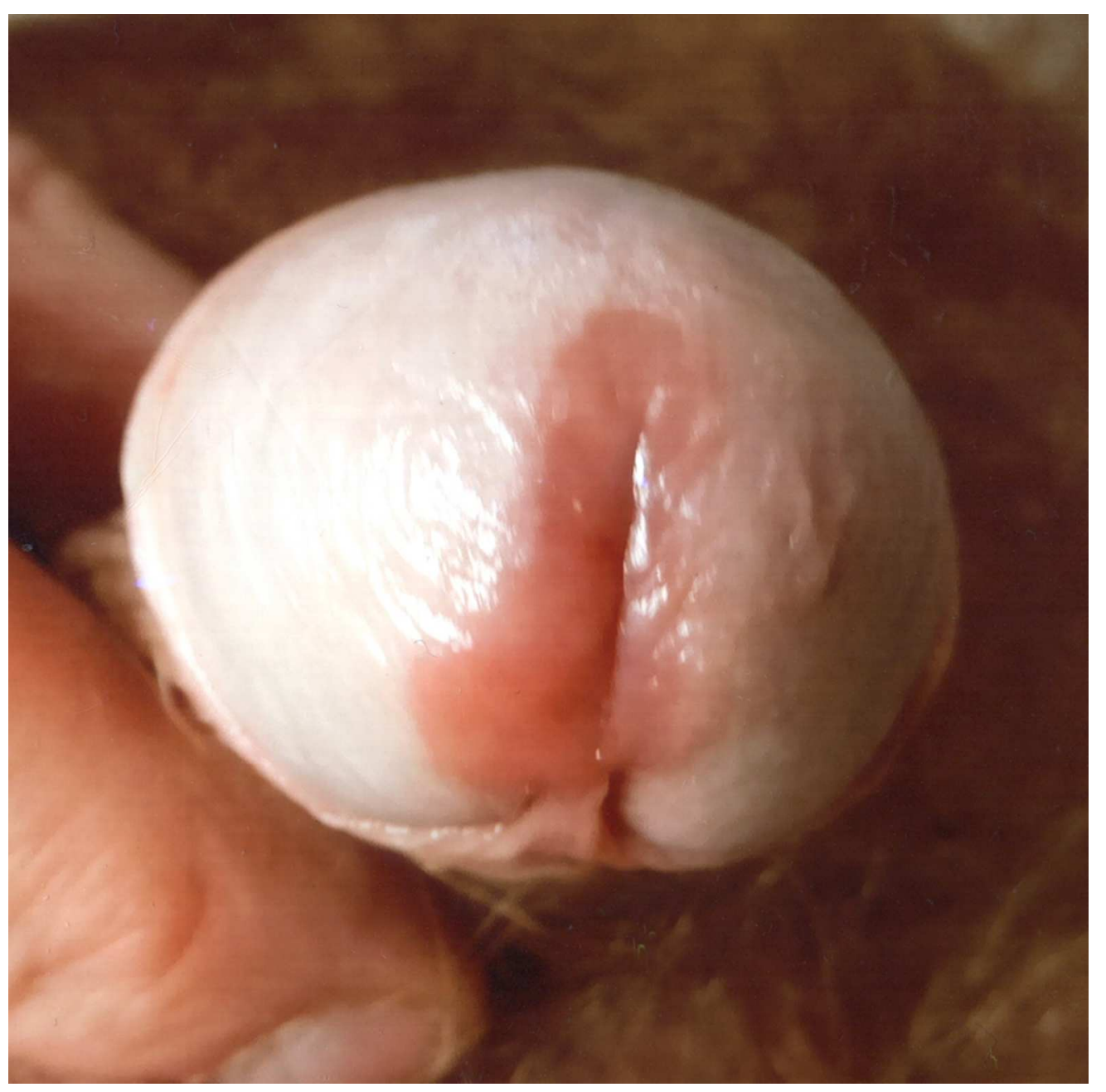

Figure 1: Peri-meatal erythematous plaque, histopathology confirmed penile intraepithelial neoplasia. A 55-year-old man presented wi $85 \times 84 \mathrm{~mm}(300 \times 300 \mathrm{DPI})$ 


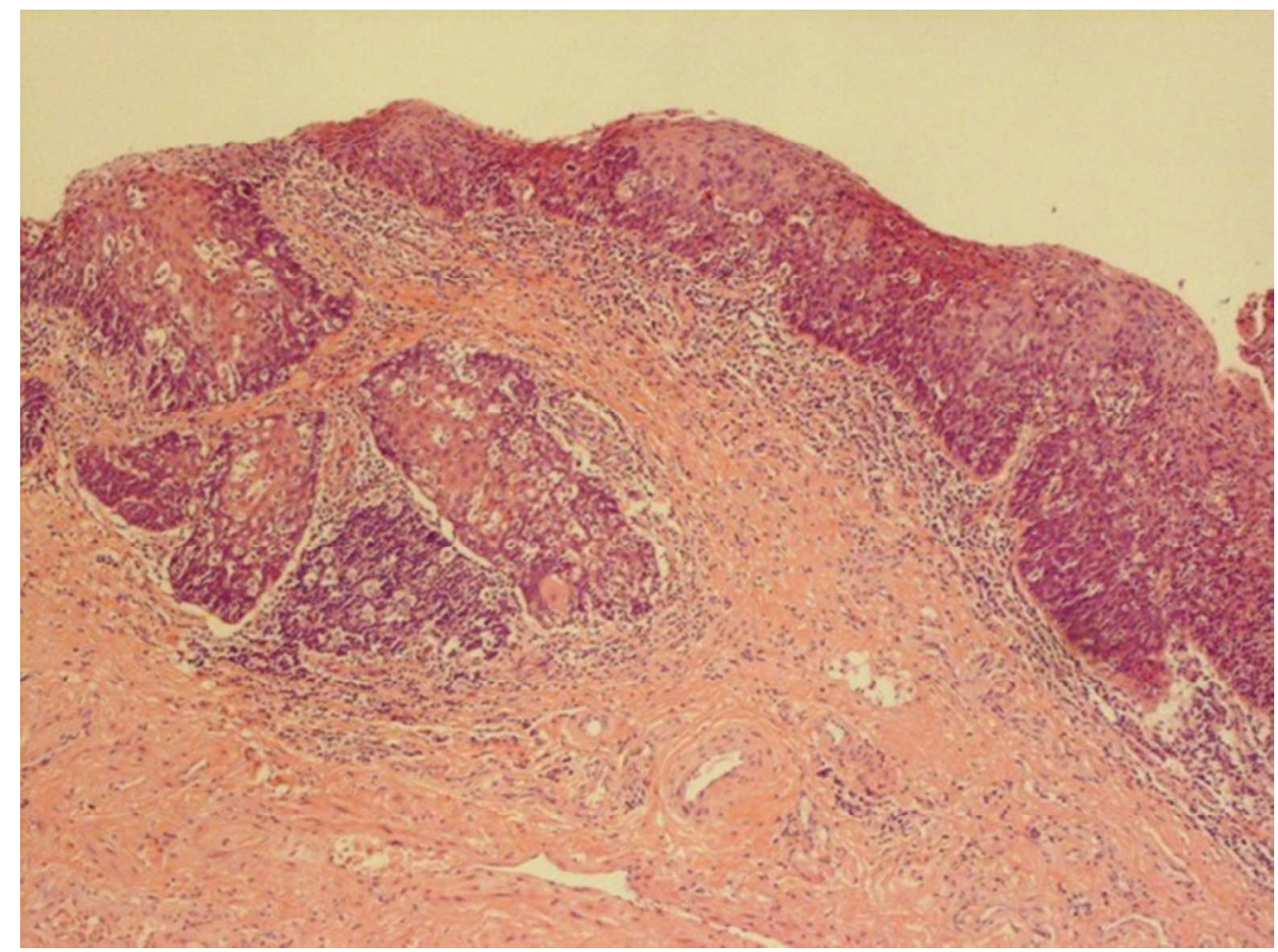

Figure 2: Invasive urethral squamous cell carcinoma (x40 magnification).

Biopsy and subsequent urethrec $216 \times 162 \mathrm{~mm}(72 \times 72 \mathrm{DPI})$ 